\title{
JORDAN STRUCTURES IN SIMPLE GRADED RINGS
}

\author{
RICHARD SPEERS
}

1. Introduction. In a recent paper [3] we proved graded analogs to theorems of Herstein [1], [2] about the Lie structure of a simple ring. In this note results about the Jordan structure of a simple graded ring will be given. The main results are Theorem 1, which states that a homogeneous Jordan ideal that contains an even element also contains an irrelevant ideal, and Theorem 2 , which states that a homogeneous Jordan ideal that is also a subring must contain an irrelevant ideal.

2. Preliminaries. In a graded ring $R=\oplus_{i \geq 0} R_{i}$, ideals of the form $\oplus_{i \geq n} R_{i}$ are irrelevant. $R$ is a simple graded ring (sgr) if $R_{i} R_{j} \neq(0)$ for all $i$ and $j$, and $R$ has no relevant homogeneous ideals. If $x \in R_{\alpha}$ and $y \in R_{\beta}$, then $[x, y]=x y-(-1)^{\alpha \beta} y x\left((x, y)=x y+(-1)^{\alpha \beta} y x\right)$ is called their Lie product (Jordan product). The center of $R$ is $Z(R)$ $=\{x:[x, y]=0$ for all $y \in R\}$.

Proposition 1. Let $R=\oplus_{i \geqq 0} R_{i}$ be a sgr. If $0 \neq a \in R_{j}$, then $R_{i} a R_{k}$ $=R_{i+j+k}$ for all $i$ and $k$. If $b$ is homogeneous and $R_{0} b R_{0}=(0)$, then $b=0$.

A proof may be found in [3].

3. Lemma. Let $R$ be a graded ring and let $U$ be a homogeneous Jordan ideal of $R$. If $a, b \in U$ are homogeneous, then for all homogeneous $x \in R$ we have $[(a, b), x] \in U$.

Proof. $(a,[x, b])-([a, x], b)=(-1)^{a x}[x,(a, b)]$. The left side of the equation is an element of $U$, so the result follows.

THEOREM 1. Let $R=\oplus_{i \geqq 0} R_{i}$ be a sgr of characteristic $\neq 2$ and let $U$ be a homogeneous Jordan ideal of $R$. If $U$ contains a nonzero even element of $R$, then $U$ contains a nonzero irrelevant ideal of $R$.

Proof. Let $a, b \in U x \in R$ be homogeneous. Then $[(a, b), x] \in U$ and $((a, b), x) \in U$ imply $2 x(a, b) \in U$ which in turn implies that $(2 x(a, b), y)$ $\in U$ for all homogeneous $y$. Thus, $2 R_{i}(a, b) R_{j} \subseteq U$ for all $i$ and $j$.

If $2 R_{i}(a, b) R_{j}=(0)$ for all $i$ and $j$, then by Proposition $1(a, b)=0$, and so in this case $(U, U)=(0)$. If $0 \neq a \in U$ is even, $0=(a,(a, x))$ implies $2 a x a=0$ for all homogeneous $x$. Thus, $a=0$, a contradiction.

Hence, there exist $i$ and $j$ such that $0 \neq 2 R_{i}(a, b) R_{j} \subseteq U$. Therefore, $U \supseteq \oplus_{k \geq a+b+i+j} R_{k} \neq(0)$.

Received by the editors April 30, 1969. 
Proposition 2. Let $R$ be $a$ sgr, char $R \neq 2$, and let $U$ be a homogeneous Jordan ideal of $R$ that does not contain a nonzero irrelevant ideal of $R$. If $a \in U$ satisfies $[a, R] \subseteq U$, then $a=0$.

Proof. Let $x$ and $y$ be homogeneous. If $a \neq 0$, then $[a, x] \in U$ and $(a, x) \in U$ imply $a x \in U$, so $(a x, y) \in U$. Thus, yax $\in U$, so $U \supseteq \oplus_{i \geqq a} R_{i}$.

Corollary. With $R$ and $U$ as above, $U \cap Z(R)=(0)$.

Theorem 2. Let $R$ be a sgr, char $R \neq 2$, and let $U$ be a homogeneous Jordan ideal and a subring of $R$. Either $U=(0)$ or $U$ contains a nonzero irrelevant ideal of $R$.

Proof. If $(U, U)=(0), a=0$ for all even $a \in U$. If $0 \neq a \in U$ is odd, $a^{2}=0$ and so $a(a, x)=0$ for all even $x \in R$. Hence, $a x a=0$, so $a=0$. Thus, $(U, U)=(0)$ implies that $U=(0)$.

If $(U, U) \neq(0)$ let $a$ and $b \in U,(a, b) \neq 0$, and let $c$ be homogeneous. Then $(a b, c)=(a, b) c-(-1)^{a b} b(a, c)+(-1)^{a(b+c)}(b, c a)$. Thus, $(a, b) R_{i} \subseteq U$ for all $i$. Let $d$ be homogeneous. Then $(d,(a, b) c)$ $=d(a, b) c+(-1)^{d(a+b+c)}(a, b) c d \in U$. Now,

$$
(a, b c d)=a b c d+(-1)^{a(b+c+d)} b c d a \in U .
$$

An examination of the parities involved shows that $(a, b c d)=(a, b) c d$ $\pm(b a, c d) \pm(c d, b) a$. Thus $(a, b) c d \in U$, and so $d(a, b) c \in U$. This implies that $U \supseteq \oplus_{i \geqq a+b} R_{i}$.

\section{REFERENCES}

1. I. N. Herstein, Lie and Jordan structures in simple, associative rings, Bull. Amer. Math. Soc. 67 (1961), 517-531. MR 25 \#3072.

2. - Topics in ring theory, University of Chicago, Chicago, Ill., 1961.

3. R. Speers, Lie structures in simple graded rings, Duke Math. J. (to appear).

Skidmore College 